\title{
Beta Generalization of Stancu-Durrmeyer Operators Involving a Generalization of Boas-Buck Type Polynomials
}

\author{
Gurhan ICOZ ${ }^{(0)}$, Hatice ERYIGIT* \\ Gazi University, Faculty of Science, Department of Mathematics, 06560, Ankara, Turkey
}

\section{Highlights}

- In this study, we used polynomials in the operator.

- The generalization mentioned in the study included a generalization of Boas-Buck type polynomials.

- The operator was established with the help of integral representation.

- The method which we used was the Stancu and Durrmeyer types generalization.

- Rate of convergence was given by modulus of continuity and Lipschitz class functions.

\section{ArticleInfo}

Received: 08/08/2019

Accepted: 25/02/2020

\section{Keywords}

Szász operators

Stancu type generalization

Beta function

\begin{abstract}
We deal with beta generalization of Stancu-Durrmeyer operators via a generalization of Boas-Buck type polynomials with the help of analytic functions. Approximation properties are investigated and convergence results are obtained for the operators.
\end{abstract}

\section{INTRODUCTION}

At last decades, the extensions of Szász operators [1] which resembles to Poisson distrubitions have attracted many researcher in the area. Using Appell and Sheffer polynomials, Jakimovski-Leviatan [2] and Ismail [3] have defined well-known generalization of Szász operators. The theory have been thrived more due to the increasing interest [4-15]. Orthogonal and d-orthogonal polynomials have been used commonly. After all, a new sequence of operators which yields a generalization of Boas-Buck type polynomials [16] is as follows

$R(v) \Psi(x S(v)+\sigma(v))=\sum_{j=0}^{\infty} \theta_{j}(x) v^{j}$,

$H_{n}(f ; x)=\frac{1}{R(1) \Psi(n x S(1)+\sigma(1))} \sum_{j=0}^{\infty} \theta_{j}(n x) f\left(\frac{j}{n}\right)$,

where $R, \Psi, S$ and $\sigma$ have formal power series representations at the disc $|z|<L(L>1)$ with the following exception

$S(v)=\sum_{j=0}^{\infty} s_{j} v^{j+1},\left(s_{0} \neq 0\right)$ 
and

$\sigma(v)=\sum_{j=0}^{\infty} \sigma_{j} v^{j+2}$

After accepting the following restrictions, the operator will be positive and convergence problem of the operators (2) will be completed:

(1) $\Psi: \mathbb{R} \rightarrow(0, \infty)$

(2) $\theta_{j}(x) \geq 0$ for $j=0,1,2, \ldots$

(3) $R(1)>0$ and $S^{\prime}(1)=1$.

Sucu and Varma [8] defined Kantorovich generalization of the operator $H_{n}^{*}$ as

$$
H_{n}^{*}(f ; x)=\frac{n}{R(1) \Psi(n x S(1)+\sigma(1))} \sum_{j=0}^{\infty} \theta_{j}(n x) \int_{\frac{j}{n}}^{\frac{j+1}{n}} f(\xi) d \xi
$$

They obtained convergence properties and estimated approximation properties of the operators.

In the present paper, we define Beta generalization of Stancu-Durrmeyer operators of (2) as follows

$$
L_{n}(f ; x)=\frac{1}{R(1) \Psi(n x S(1)+\sigma(1))} \sum_{k=0}^{\infty} \frac{\theta_{k}(n x)}{B(n+1, k)} \int_{0}^{\infty} \frac{t^{k-1}}{(1+t)^{n+k+1}} f\left(\frac{n t+\alpha}{n+\beta}\right) d t
$$

where $x \in[0, \infty)$ and $f \in \mathrm{C}[0, \infty)$. We assume that the restriction is valid. Also, $S(v)$ and $\sigma(v)$ are given by (3) and (4), respectively. Beta integral is defined by

$$
B(n, k)=\int_{0}^{\infty} \frac{t^{k-1}}{(1+t)^{n+k}} d t=\frac{\Gamma(\mathrm{n}) \Gamma(\mathrm{k})}{\Gamma(\mathrm{n}+\mathrm{k})}
$$

which $\Gamma$ is well known Gamma function and $n, k>0$.

\section{MAIN RESULTS}

Firstly, some equalities are obtained for $L_{n}$.

Lemma 1. When we use (1), then we easily get equations as follows:

$$
\begin{aligned}
& L_{n}(1 ; x)=1, \\
& L_{n}(\zeta ; x)=\frac{n}{n+\beta} \frac{\Psi^{\prime}(n x S(1)+\sigma(1))}{\Psi(n x S(1)+\sigma(1))} x+\frac{1}{n+\beta}\left(\frac{R^{\prime}(1)}{R(1)}+\frac{\Psi^{\prime}(n x S(1)+\sigma(1))}{\Psi(n x S(1)+\sigma(1))} \sigma^{\prime}(1)+\alpha\right), \\
& L_{n}\left(\zeta^{2} ; x\right)=\frac{n^{3}}{(n+\beta)^{2}(n-1)} \frac{\Psi^{\prime \prime}(n x S(1)+\sigma(1))}{\Psi(n x S(1)+\sigma(1))} x^{2}+ \\
& \frac{n^{2}}{(n+\beta)^{2}(n-1)}\left\{\frac{\left[2 R^{\prime}(1)+R(1)\left(2+S^{\prime \prime}(1)\right)\right] \Psi^{\prime}(n x S(1)+\sigma(1))+2 R(1) \sigma^{\prime}(1) \Psi^{\prime \prime}(n x S(1)+\sigma(1))}{R(1) \Psi(n x S(1)+\sigma(1))}+\right. \\
& \left.\frac{2 \alpha(n-1)}{n} \frac{\Psi^{\prime}(n x S(1)+\sigma(1))}{\Psi(n x S(1)+\sigma(1))}\right\} x+\frac{n}{(n+\beta)^{2}(n-1)}\left\{\frac{\left(\sigma^{\prime}(1)\right)^{2} R^{\prime}(1) \Psi^{\prime \prime}(n x S(1)+\sigma(1))}{R(1) \Psi(n x S(1)+\sigma(1))}\right\}+
\end{aligned}
$$


$\frac{n}{(n+\beta)^{2}(n-1)}\left\{\frac{\left[\left(2 R^{\prime}(1)+R(1)\right) \sigma^{\prime}(1)+R(1)\left(\sigma^{\prime \prime}(1)+\sigma^{\prime}(1)\right)\right] \Psi^{\prime}(n x S(1)+\sigma(1))}{R(1) \Psi(n x S(1)+\sigma(1))}+\frac{R^{\prime \prime}(1)+2 R^{\prime}(1)}{R(1)}\right\}+$ $+\frac{2 \alpha}{(n+\beta)^{2}}\left(\frac{R^{\prime}(1)}{R(1)}+\frac{\Psi^{\prime}(n x S(1)+\sigma(1))}{\Psi(n x S(1)+\sigma(1))} \sigma^{\prime}(1)\right)+\frac{\alpha^{2}}{(n+\beta)^{2}}$

Proof. If we write $v=1$ and $x \rightarrow n x$ in (1), then we get

$$
\sum_{k=0}^{\infty} \theta_{k}(n x)=R(1) \Psi(n x S(1)+\sigma(1))
$$

We rewrite the operator $L_{n}$ using the equation, we find that

$$
\begin{gathered}
L_{n}(1 ; x)=\frac{1}{R(1) \Psi(n x S(1)+\sigma(1))} \sum_{k=0}^{\infty} \frac{\theta_{k}(n x)}{B(n+1, k)} \int_{0}^{\infty} \frac{t^{k-1}}{(1+t)^{n+k+1}} d t \\
=\frac{1}{R(1) \Psi(n x S(1)+\sigma(1))} \sum_{k=0}^{\infty} \frac{\theta_{k}(n x)}{B(n+1, k)} B(n+1, k) \\
=\frac{1}{R(1) \Psi(n x S(1)+\sigma(1))} \sum_{k=0}^{\infty} \theta_{k}(n x)=1 .
\end{gathered}
$$

Now, we derivate the operator with respect to $v$ in (1), then we take $v=1$ and $x \rightarrow n x$, it is clearly seen that

$\sum_{k=0}^{\infty} k \theta_{k}(n x)=R^{\prime}(1) \Psi(n x S(1)+\sigma(1))+R(1) \Psi^{\prime}(n x S(1)+\sigma(1))\left[n x S^{\prime}(1)+\sigma^{\prime}(1)\right]$.

Thus, we have the equation (9).

Lastly, we take second derivative of (1) with respect to $v$ and then we take $v=1$ and $x \rightarrow n x$ in there, we have the below form

$$
\begin{aligned}
\sum_{k=0}^{\infty} k^{2} \theta_{k}(n x)= & {\left[R^{\prime \prime}(1)+R^{\prime}(1)\right] \Psi(n x S(1)+\sigma(1)) } \\
& +\left[2 R^{\prime}(1)+R(1)\right] \Psi^{\prime}(n x S(1)+\sigma(1))\left[n x S^{\prime}(1)+\sigma^{\prime}(1)\right] \\
& +R(1) \Psi^{\prime \prime}(n x S(1)+\sigma(1))\left[n x S^{\prime}(1)+\sigma^{\prime}(1)\right]^{2} \\
& +R(1) \Psi^{\prime}(n x S(1)+\sigma(1))\left[n x S^{\prime \prime}(1)+\sigma^{\prime \prime}(1)\right] .
\end{aligned}
$$

Hence, we get the equation (10).

Lemma 2. From (8), (9) and (10), we have

$$
\begin{aligned}
L_{n}(\zeta-x ; x)= & \frac{n}{n+\beta}\left(\frac{\Psi^{\prime}(n x S(1)+\sigma(1))}{\Psi(n x S(1)+\sigma(1))}-\frac{n+\beta}{n}\right) x \\
& +\frac{1}{n+\beta}\left(\frac{R^{\prime}(1)}{R(1)}+\frac{\Psi^{\prime}(n x S(1)+\sigma(1))}{\Psi(n x S(1)+\sigma(1))} \sigma^{\prime}(1)+\alpha\right)
\end{aligned}
$$


and

$$
\begin{aligned}
& L_{n}\left((\zeta-x)^{2} ; x\right) \\
& =\left[\frac{n^{3}}{(n+\beta)^{2}(n-1)} \frac{\Psi^{\prime \prime}(n x S(1)+\sigma(1))}{\Psi(n x S(1)+\sigma(1))}-\frac{2 n}{n+\beta} \frac{\Psi^{\prime}(n x S(1)+\sigma(1))}{\Psi(n x S(1)+\sigma(1))}+1\right] x^{2} \\
& +\frac{n}{(n+\beta)^{2}(n-1)}\left\{\frac{\left[2 R^{\prime}(1)+R(1)\left(2+S^{\prime \prime}(1)\right)\right] \Psi^{\prime}(n x S(1)+\sigma(1))}{R(1) \Psi(n x S(1)+\sigma(1))}\right. \\
& +\frac{2 R(1) \sigma^{\prime}(1) \Psi^{\prime \prime}(n x S(1)+\sigma(1))}{R(1) \Psi(n x S(1)+\sigma(1))}+\frac{2 \alpha(n-1)}{n} \frac{\Psi^{\prime}(n x S(1)+\sigma(1))}{\Psi(n x S(1)+\sigma(1))} \\
& \left.-\frac{2(n+\beta)(n-1)}{n}\left(\frac{R^{\prime}(1)}{R(1)}+\frac{\Psi^{\prime}(n x S(1)+\sigma(1))}{\Psi(n x S(1)+\sigma(1))} \sigma^{\prime}(1)+\alpha\right)\right\} x \\
& +\frac{n}{(n+\beta)^{2}(n-1)}\left\{\frac{\left(\sigma^{\prime}(1)\right)^{2} R^{\prime}(1) \Psi^{\prime \prime}(n x S(1)+\sigma(1))}{R(1) \Psi(n x S(1)+\sigma(1))}\right\} \\
& +\frac{n}{(n+\beta)^{2}(n-1)}\left\{\frac{\left[\left(2 R^{\prime}(1)+R(1)\right) \sigma^{\prime}(1)+R(1)\left(\sigma^{\prime \prime}(1)+\sigma^{\prime}(1)\right)\right] \Psi^{\prime}(n x S(1)+\sigma(1))}{R(1) \Psi(n x S(1)+\sigma(1))}\right. \\
& \left.+\frac{R^{\prime \prime}(1)+2 R^{\prime}(1)}{R(1)}\right\}+\frac{2 \alpha}{(n+\beta)^{2}}\left(\frac{R^{\prime}(1)}{R(1)}+\frac{\Psi^{\prime}(n x S(1)+\sigma(1))}{\Psi(n x S(1)+\sigma(1))} \sigma^{\prime}(1)\right)+\frac{\alpha^{2}}{(n+\beta)^{2}} .
\end{aligned}
$$

We assume that

$\lim _{y \rightarrow \infty} \frac{\Psi^{\prime \prime}(y)}{\Psi(y)}=1, \lim _{y \rightarrow \infty} \frac{\Psi^{\prime}(y)}{\Psi(y)}=1$

equalities are implemented throughout the paper.

The next theorem will show us the uniform convergence of the operator $L_{n}$.

Theorem 1. $f$ belongs to $C[0, \infty) \cap \Omega$ where

$$
\Omega=\left\{f: \frac{f(x)}{1+x^{2}} \rightarrow A\right\}
$$

Then, $\left(L_{n}\right)$ converge uniformly to $f$ on the compact subsets of each $[0, \infty]$.

Proof. One can easily see that from Lemma 1

$$
\lim _{n \rightarrow \infty} L_{n}\left(\zeta^{i} ; x\right)=x^{i}, \quad i=0,1,2
$$

It shows that the convergences are provided uniformly on the compact subsets of $[0, \infty)$. So, the proof is done with the help of Korovkin theorem [17].

For the next theorem, we will obtain approximation properties of $\left(L_{n}\right)$ using modulus of continuity.

Theorem 2. For $f$ is uniform continuous on $[0, \infty)$, we have 


$$
\left|L_{n}(f ; x)-f(x)\right| \leq 2 \omega\left(f, \sqrt{L_{n}\left((\zeta-x)^{2} ; x\right)}\right)
$$

where the modulus of continuity of $f[18]$ is showen by $\omega$ defined by

$$
\omega(f ; \delta):=\sup \{|f(x)-f(y)|:|x-y|<\delta\} .
$$

Proof. Using properties of $\omega(f ; \delta)$, we have

$$
\begin{aligned}
&\left|L_{n}(f ; x)-f(x)\right| \mid \frac{1}{R(1) \Psi(n x S(1)+\sigma(1))} \sum_{k=0}^{\infty} \frac{\theta_{k}(n x)}{B(n+1, k)} \int_{0}^{\infty} \frac{t^{k-1}}{(1+t)^{n+k+1}}\left(f\left(\frac{n t+\alpha}{n+\beta}\right)\right. \\
&-f(x)) d t \mid \\
& \leq \frac{1}{R(1) \Psi(n x S(1)+\sigma(1))} \sum_{k=0}^{\infty} \frac{\theta_{k}(n x)}{B(n+1, k)} \\
& \leq \omega(f ; \delta) \frac{t^{k-1}}{R(1+t)^{n+k+1}}\left|f\left(\frac{n t+\alpha}{n+\beta}\right)-f(x)\right| \mathrm{dt} \\
& \int_{0}^{\infty} \frac{t^{k-1}}{(1+t)^{n+k+1}}\left(1+\frac{1}{n+\beta}-x \mid\right. \\
& \leq \omega(f ; \delta)\left(1+\frac{1}{\delta} \frac{\theta_{k}(n x)}{R(1) \Psi(n x S(1)+\sigma(1))}\right) \\
& \sum_{k=0}^{\infty} \frac{\theta_{k}(n x)}{B(n+1, k)} \int_{0}^{\infty} \frac{t^{k-1}}{(1+t)^{n+k+1}}\left|\frac{n t+\alpha}{n+\beta}-x\right| d t .
\end{aligned}
$$

Applying the Cauchy-Schwarz inequality for the above integral, we get

$$
\begin{aligned}
& \int_{0}^{\infty} \frac{t^{k-1}}{(1+t)^{n+k+1}}\left|\frac{n t+\alpha}{n+\beta}-x\right| d t \\
& \leq \sqrt{\int_{0}^{\infty} \frac{t^{k-1}}{(1+t)^{n+k+1}}} \sqrt{\int_{0}^{\infty} \frac{t^{k-1}}{(1+t)^{n+k+1}}\left(\frac{n t+\alpha}{n+\beta}-x\right)^{2} d t} \\
& =\sqrt{B(n+1, k)} \sqrt{\int_{0}^{\infty} \frac{t^{k-1}}{(1+t)^{n+k+1}}\left(\frac{n t+\alpha}{n+\beta}-x\right)^{2} d t .}
\end{aligned}
$$

Now, we use Cauchy-Schwarz inequality for the sum, we see that 


$$
\begin{aligned}
& \frac{1}{R(1) \Psi(n x S(1)+\sigma(1))} \sum_{k=0}^{\infty} \frac{\theta_{k}(n x)}{B(n+1, k)} \int_{0}^{\infty} \frac{t^{k-1}}{(1+t)^{n+k+1}}\left|\frac{n t+\alpha}{n+\beta}-x\right| d t \\
& \leq \frac{1}{R(1) \Psi(n x S(1)+\sigma(1))} \sum_{k=0}^{\infty} \frac{\theta_{k}(n x)}{\sqrt{B(n+1, k)}} \sqrt{\int_{0}^{\infty} \frac{t^{k-1}}{(1+t)^{n+k+1}}\left(\frac{n t+\alpha}{n+\beta}-x\right)^{2} d t} \\
& =\sum_{k=0}^{\infty} \sqrt{\frac{\theta_{k}(n x)}{R(1) \Psi(n x S(1)+\sigma(1))}} \sqrt{\frac{\theta_{k}(n x)}{R(1) \Psi(n x S(1)+\sigma(1)) B(n+1, k)} \int_{0}^{\infty} \frac{t^{k-1}}{(1+t)^{n+k+1}}\left(\frac{n t+\alpha}{n+\beta}-x\right)^{2} d t}
\end{aligned}
$$

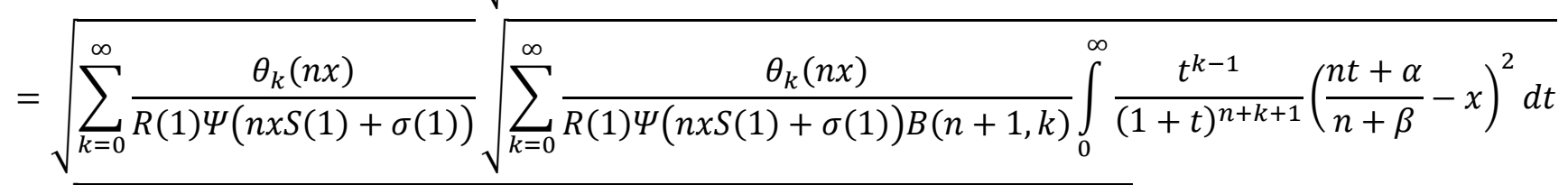

$$
\begin{aligned}
& =\sqrt{\frac{1}{R(1) \Psi(n x S(1)+\sigma(1))} \sum_{k=0}^{\infty} \frac{\theta_{k}(n x)}{B(n+1, k)} \int_{0}^{\infty} \frac{t^{k-1}}{(1+t)^{n+k+1}}\left(\frac{n t+\alpha}{n+\beta}-x\right)^{2} d t} \\
& =\sqrt{L_{n}\left((\zeta-x)^{2} ; x\right)} \text {. }
\end{aligned}
$$

Considering this inequality in (13), we find

$$
\left|L_{n}(f ; x)-f(x)\right| \leq\left(1+\frac{1}{\delta} \sqrt{L_{n}\left((\zeta-x)^{2} ; x\right)}\right) \omega(f ; \delta) .
$$

If we take $\delta=\sqrt{L_{n}\left((\zeta-x)^{2} ; x\right)}$, then the desired result is hold.

Now, we recall the Lipschitz class functions for $0<\tau \leq 1$ and $\lambda_{1}, \lambda_{2} \in[0, \infty)$

$$
\operatorname{Lip}_{R}^{(\tau)}:=\left\{\Phi:\left|\Phi\left(\lambda_{1}\right)-\Phi\left(\lambda_{2}\right)\right| \leq R\left|\lambda_{1}-\lambda_{2}\right|^{\tau}\right\}
$$

Theorem 3. For $\Phi \in \operatorname{Lip}_{R}^{(\tau)}$, we have

$$
\left|L_{n}(\Phi ; x)-\Phi(x)\right| \leq R\left[L_{n}\left((\zeta-x)^{2} ; x\right)\right]^{\tau / 2}
$$

Proof. We exert $\Phi \in \operatorname{Lip}_{R}^{(\tau)}$ to the operator $L_{n}$

$$
\begin{aligned}
\mid L_{n}(\Phi ; x)- & \Phi(x)|=| L_{n}(\Phi(\zeta)-\Phi(x) ; x) \mid \\
& \leq L_{n}(|\Phi(\zeta)-\Phi(x)| ; x) \leq R L_{n}\left(|\zeta-x|^{\tau} ; x\right) .
\end{aligned}
$$

Using Hölder inequality for the integrals, we obtain 


$$
\begin{aligned}
\int_{0}^{\infty} \frac{t^{k-1}}{(1+t)^{n+k+1}} & \left|\frac{n t+\alpha}{n+\beta}-x\right|^{\tau} d t=\int_{0}^{\infty}\left(\frac{t^{k-1}}{(1+t)^{n+k+1}}\right)^{\frac{2-\tau}{2}}\left(\frac{t^{k-1}}{(1+t)^{n+k+1}}\right)^{\frac{\tau}{2}}\left|\frac{n t+\alpha}{n+\beta}-x\right|^{\tau} d t \\
& \leq\left(\int_{0}^{\infty} \frac{t^{k-1}}{(1+t)^{n+k+1}}\right)^{\frac{2-\tau}{2}}\left(\int_{0}^{\infty} \frac{t^{k-1}}{(1+t)^{n+k+1}}\left(\frac{n t+\alpha}{n+\beta}-x\right)^{2} d t\right)^{\frac{\tau}{2}} \\
& \leq(B(n+1, k))^{\frac{2-\tau}{2}}\left(\int_{0}^{\infty} \frac{t^{k-1}}{(1+t)^{n+k+1}}\left(\frac{n t+\alpha}{n+\beta}-x\right)^{2} d t\right)^{\frac{\tau}{2}} .
\end{aligned}
$$

Using Hölder inequality for the sum, we attain

$$
\begin{aligned}
& \frac{1}{R(1) \Psi(n x S(1)+\sigma(1))} \sum_{k=0}^{\infty} \frac{\theta_{k}(n x)}{B(n+1, k)} \int_{0}^{\infty} \frac{t^{k-1}}{(1+t)^{n+k+1}}\left|\frac{n t+\alpha}{n+\beta}-x\right|^{\tau} d t \leq \\
& \sum_{k=0}^{\infty}\left(\frac{\theta_{k}(n x)}{R(1) \Psi(n x S(1)+\sigma(1))}\right)^{\frac{2-\tau}{2}}\left(\frac{\theta_{k}(n x)}{R(1) \Psi(n x S(1)+\sigma(1)) B(n+1, k)} \int_{0}^{\infty} \frac{t^{k-1}}{(1+t)^{n+k+1}}\left(\frac{n t+\alpha}{n+\beta}-x\right)^{2}\right)^{\frac{\tau}{2}} d t \leq \\
& \left(\sum_{k=0}^{\infty} \frac{\theta_{k}(n x)}{R(1) \Psi(n x S(1)+\sigma(1))}\right)^{\frac{2-\tau}{2}} \times\left(\frac{1}{R(1) \Psi(n x S(1)+\sigma(1))} \sum_{k=0}^{\infty} \frac{\theta_{k}(n x)}{B(n+1, k)} \int_{0}^{\infty} \frac{t^{k-1}}{(1+t)^{n+k+1}}\left(\frac{n t+\alpha}{n+\beta}-x\right)^{2} d t\right)^{\frac{\tau}{2}}= \\
& \left(L_{n}\left((\zeta-x)^{2} ; x\right)\right)^{\frac{\tau}{2}} .
\end{aligned}
$$

From the inequality it becomes (14). So, the proof is done.

Firstly, we recall Gavrea and Raşa's result [19] and second order Steklov function where will use to give quantitative estimation for the operator (2).

Let $z \in C^{2}[0, a]$ and $\left(L_{n}\right)_{n \geq 0}$ be a sequence of linear positive operators with the property $L_{n}\left(e_{i} ; x\right)=$ $e_{i}(x), e_{i}(\zeta)=\zeta^{i}, i=0,1,2$. Then, Raşa's result is known as

$$
\left|L_{n}(z ; x)-z(x)\right| \leq\left\|z^{\prime}\right\| \sqrt{L_{n}\left((\zeta-x)^{2} ; x\right)}+\frac{1}{2}\left\|z^{\prime \prime}\right\| L_{n}\left((\zeta-x)^{2} ; x\right)
$$

For $f \in C[a, b]$, the second order Steklov function of $f$ is defined by

$$
f_{h}(x):=\frac{1}{h} \int_{-h}^{h}\left(1-\frac{|t|}{h}\right) f(h ; x+t) d t, x \in[a, b],
$$

where $f(h ;):.[a-h, b+h] \rightarrow \mathbb{R}, h>0$, by

$$
f(h ; x)=\left\{\begin{array}{ccc}
P_{-}(x) & ; \quad a-h \leq x<a \\
f(x) & ; \quad a \leq x \leq b \\
P_{+}(x) & ; \quad b<x \leq b+h
\end{array}\right.
$$

and $P_{-}, P_{+}$are the best linear approximations to $f$ on the indicated intervals.

Theorem 4. Let $\Phi$ be a continuous function on $[0, \infty)$. Then, it holds that 


$$
\left|L_{n}(\Phi ; x)-\Phi(x)\right| \leq \frac{3}{2}\left(1+\frac{a}{2}+\frac{h^{2}}{2}\right) \omega_{2}(\Phi ; h)+\frac{2 h^{2}}{a}\|\Phi\|
$$

where $\omega_{2}$ is the second order modulus of continuity of the function $\Phi[18]$ defined by

$$
\omega_{2}(\Phi ; h):=\sup _{0<t<h}\|\Phi(.+2 t)-2 \phi(.+t)+\Phi(.)\| .
$$

Proof. From simple algebraic computations, we set

$$
L_{n}(\Phi ; x)-\Phi(x)=L_{n}\left(\Phi-\Phi_{h} ; x\right)+L_{n}\left(\Phi_{h} ; x\right)-\Phi_{h}(x)+\Phi_{h}(x)-\Phi(x)
$$

and triangle inequality we reach

$$
\begin{aligned}
\left|L_{n}(\Phi ; x)-\Phi(x)\right| & \leq L_{n}\left(\left|\Phi-\Phi_{h}\right| ; x\right)+\left|L_{n}\left(\Phi_{h} ; x\right)-\Phi_{h}(x)\right|+\left|\Phi_{h}(x)-\Phi(x)\right| \\
& \leq 2\left\|\Phi-\Phi_{h}\right\|+\left|L_{n}\left(\Phi_{h} ; x\right)-\Phi_{h}(x)\right|
\end{aligned}
$$

where $\Phi_{h}$ is the second order Steklov function of $\Phi$ [19]. It is known that $\Phi_{h} \epsilon C^{2}[0, a]$. In view of this fact, we obtained the following expression by Raşa's result [19] and Landau inequality

$$
\begin{gathered}
\left|L_{n}\left(\Phi_{h} ; x\right)-\Phi_{h}(x)\right| \leq\left\|\Phi_{h}{ }^{\prime}\right\| \sqrt{L_{n}\left((\zeta-x)^{2} ; x\right)}+\frac{1}{2}\left\|\Phi_{h}{ }^{\prime \prime}\right\| L_{n}\left((\zeta-x)^{2} ; x\right) \\
\leq\left(\frac{2}{a}\left\|\Phi_{h}\right\|+\frac{a}{2}\left\|\Phi_{h}{ }^{\prime \prime}\right\|\right) \sqrt{L_{n}\left((\zeta-x)^{2} ; x\right)}+\frac{1}{2}\left\|\Phi_{h}{ }^{\prime \prime}\right\| L_{n}\left((\zeta-x)^{2} ; x\right) \\
\leq\left(\frac{2}{a}\|\Phi\|+\frac{3 a}{4} \frac{1}{h^{2}} \omega_{2}(\Phi ; h)\right) \sqrt{L_{n}\left((\zeta-x)^{2} ; x\right)} \\
+\frac{3}{4} \frac{1}{h^{2}} \omega_{2}(\Phi ; h) L_{n}\left((\zeta-x)^{2} ; x\right) .
\end{gathered}
$$

According to Zhuk [20], there is a connection between the second order Steklov function and $\omega_{2}(\Phi ; h)$ as follows

$$
\left\|\Phi-\Phi_{h}\right\| \leq \frac{3}{4} \omega_{2}(\Phi ; h) .
$$

Using this inequality and (16) in (15) give us

$$
\begin{aligned}
\left|L_{n}(\Phi ; x)-\Phi(x)\right| & \leq \frac{3}{2} \omega_{2}(\Phi ; h)+\left(\frac{2}{a}\|\Phi\|+\frac{3 a}{4 h^{2}} \omega_{2}(\Phi ; h)\right) h^{2}+\frac{3}{4} \frac{1}{h^{2}} \omega_{2}(\Phi ; h) h^{4} \\
= & \frac{3}{2}\left(1+\frac{a}{2}+\frac{h^{2}}{2}\right) \omega_{2}(\Phi ; h)+\frac{2 h^{2}}{a}\|\Phi\|,
\end{aligned}
$$

where $h=\sqrt[4]{L_{n}\left((\zeta-x)^{2} ; x\right)}$.

\section{CONCLUSION}

After reading the paper, one can generalize the operator with the same mining. Authors can see the configuration how to use polynomials in the operators. They can extend the theory with using different 
polynomials in the operators. Also, they find out new generalization or new formula for the polynomials. They can compare the operators, approximation properties and rate of convergences via graphs of the operators. They can work in new spaces where theory is valid. They develope the rate of convergence.

\section{CONFLICTS OF INTEREST}

No conflict of interest was declared by the authors.

\section{REFERENCES}

[1] Szász, O., “Generalization of S. Bernstein's polynomials to the infinite interval”, Journal of Research of the National Bureau of Standarde, 45: 239-245, (1950).

[2] Jakimovski, A., Leviatan, D., "Generalized Szász operators for the approximation in the infinite interval”, Mathematica, 11: 97-103, (1969).

[3] Ismail, M.E.H.,“On a generalization of Szász operators”, Mathematica, 39: 259-267, (1974).

[4] Varma, S., Sucu, S.,I̧çöz, G., "Generalization of Szász operators involving Brenke type polynomials", Computers and Mathematics with Applications, 64: 121-127, (2012).

[5] Sucu, S., İçöz, G., Varma, S., "On some extensions of Szász operators including Boas-Buck-type polynomials", Abstract and Applied Analysis, (2012).

[6] İçöz, G., Varma, S., Sucu, S., "Approximation by operators including generalized Appell polynomials", Filomat, 30: 429-440, (2016).

[7] İçöz, G., Çekim, B., "Stancu-type generalizations of the Chan-Chyan-Srivastava operators", Filomat, 30: 3733-3742, (2016).

[8] Sucu, S., Varma, S., "Approximation by sequence of operators involving analytic functions", Mathematics, 7(2): 188, (2019).

[9] Sucu, S., Varma, S., "Generalization of Jakimovski-Leviatan type Szász operators", Applied Mathematics and Computation, 270: 977-983, (2015).

[10] Sucu, S., "Dunkl analogue of Szász operators", Applied Mathematics and Computation, 244: 42$48,(2014)$.

[11] Cai, Q. B., Yüksel, İ., Dinlemez Kantar, Ü., Çekim, B., “Approximation Properties of Durrmeyer Type of-Bleimann-Butzer and Hahn Operators", Journal of Function Spaces. Volume 2019, Article ID 7047656, 11 pages (2019).

[12] Çekim, B., Dinlemez Kantar, Ü., Yüksel, İ., "Dunkl generalization of Szász Beta-type operators”, Mathematical Methods in the Applied Sciences, 40 (18): 7697-7704, (2017).

[13] Yazıcı, S., Çekim, B., “A Kantorovich type generalization of the Szász operators via two variable Hermite polynomials", Gazi University Journal of Science, 30(4): 432-440, (2017).

[14] Taşdelen, F., Söylemez, D., Aktaş, R., "Dunkl-Gamma type operators including Appell polynomials", Complex Analysis and Operator Theory, 13(7): 3359-3371, (2019). 
[15] Aktaş, R., Söylemez, D., Taşdelen, F., "Stancu Type Generalization of Szász-Durrmeyer Operators Involving Brenke-Type Polynomials", Filomat, 33(3), (2019).

[16] Rainville, E.D., "Special Functions”, Macmillan, New York, NY, USA, (1960).

[17] Altomare, F., Campiti, M., "Korovkin-Type Approximation Theory and Its Applications", Appendix A by Michael Pannenberg and Appendix B by Ferdinand Beckhoff, de Gruyter Studies in Mathematics, 17; Walter de Gruyter \& Co.: Berlin, Germany, (1994).

[18] Devore, R.A., Lorentz, G.G., “Constructive Approximation”, Springer, Berlin, Germany, (1993).

[19] Gavrea, I.,Raşa, I., "Remarks on some quantitative Korovkin-type results", Rev.Anal. Numér. Théor. Approx., 22(2): 173-176, (1993).

[20] Zhuk, V.V., "Functions of the Lip1 class and S. N. Bernstein's polynomials (Russian)", Vestnik Leningradskogo Universiteta. Matematika, Mekhanika, Astronomiya. Leningrad Univ., Leningrad., 1: 25-30, (1989). 